\title{
CRIAÇÃO DO PNEUMOPERITÔNIO MEDIANTE PUNÇÃO COM AGULHA DE VERESS NO HIPOCÔNDRIO ESQUERDO: ENSAIO CLÍNICO, PROSPECTIVO E RANDOMIZADO
}

\author{
PNEUMOPERITONEUM BY USING A VERESS NEEDLE PUNCTURE IN THE LEFT \\ HYPOCHONDRIAC REGION: A PROSPECTIVE, RANDOMIZED CLINICAL TRIAL
}

\author{
Otávio Cansanção de Azevedoㅜ; João Luiz Moreira Coutinho Azevedo, ECBC-SP²; \\ Albino Augusto Sorbello; Antônio Cláudio de Godoy ${ }^{4}$; \\ Francisco Julimar Correia de Menezes; ${ }^{5}$ Gilmara da Silva Aguiar ${ }^{6}$
}

\begin{abstract}
RESUMO: Objetivo: Na criação do pneumoperitônio com agulha de Veress por punção na linha média do abdome têm sido relatados acidentes que, apesar de raros, afetam freqüentemente os grandes vasos. O objetivo desta pesquisa é testar a eficácia da punção alternativa no hipocôndrio esquerdo e avaliar a eficácia e segurança desta punção. Método: Sessenta e dois pacientes distribuídos aleatoriamente em dois grupos foram estudados prospectivamente: grupo HE, punção no hipocôndrio esquerdo $(\mathrm{n}=30)$, e grupo LM, punção na linha média do abdome $(n=32)$. Foram avaliados os testes de posicionamento da agulha, o número de tentativas frustradas e a duração da instalação do pneumoperitônio. Os fluxos correntes, as pressões intraperitoneais e os volumes injetados foram registrados a cada 20 segundos, até $12 \mathrm{mmHg}$. Resultados: Os testes de posicionamento foram positivos em ambos os grupos. Ocorreram duas tentativas infrutíferas de punção no grupo LM e uma no grupo HE. O tempo de duração para o estabelecimento do pneumoperitônio foi em média de 3 minutos e 46 segundos para o Grupo HE e de 4 minutos e 2 segundos para o grupo LM. As médias dos fluxos, das pressões e dos volumes foram respectivamente equivalentes entre os grupos. A análise estatística demonstrou que a punção no HE foi tão eficaz no estabelecimento do pneumoperitônio quanto a punção na LM do abdome. Conclusões: A punção no HE deve ser o local de escolha para a punção com agulha de Veress na criação do pneumoperitônio artificial pela técnica fechada, por ser segura e eficaz (Rev. Col. Bras. Cir. 2005; 32(5): 273-278).
\end{abstract}

Descritores: Laparoscopia/ efeitos adversos; Procedimentos cirúrgicos operatórios; Pneumoperitônio artificial; Biópsia por agulha; Biópsia por agulha/ efeitos adversos.

\section{INTRODUÇÃO}

A criação do pneumoperitônio é o primeiro passo para a realização da videolaparoscopia. A maioria das complicações associadas a esse procedimento ocorre durante a sua etapa mais crítica, o acesso à cavidade peritoneal ${ }^{1}$, em razão do risco significativo de lesões vasculares e viscerais ${ }^{2}$. Ferimentos vasculares representam a causa mais comum de morte em procedimentos laparoscópicos $(15 \%)^{3}$. Danos em grandes vasos podem ocorrer quando a agulha de Veress é inserida às cegas no abdome, antes da insuflação, como ocorre na técnica fechada ${ }^{3}$. Apesar de não existir consenso quan- to ao melhor método para o acesso à cavidade peritoneal visando à instauração do pneumoperitônio ${ }^{4}$, a punção com agulha de Veress ${ }^{5}$ é a técnica mais freqüentemente utilizada ${ }^{2,6}$. Estudo considerando 155.987 procedimentos laparoscópicos, em $81 \%$ foi utilizada a agulha de Veress ${ }^{6}$.

O local clássico da punção com agulha de Veress é a linha média do abdome, junto à cicatriz umbilical ${ }^{7}$. Nessa região, a punção apresenta riscos de lesão de grandes vasos, em função da pequena distância da parede anterior do abdome com essas estruturas vasculares retroperitoneais. Em pessoas magras, essa distância pode ser menor que dois centímetros $^{3}$. A aorta abdominal e a veia cava inferior, assim como

1. Médico-Assistente do Serviço de Gastroenterologia Cirúrgica do Hospital do Servidor Público do Estado de São Paulo; Mestrando do Programa de Pós-Graduação em Cirurgia e Experimentação da Universidade Federal de São Paulo (UNIFESP); Pesquisador do Grupo de Videocirurgia do CNPq/ UNIFESP.

2. Professor Adjunto, Mestre e Doutor do Departamento de Cirurgia e Responsável pelo Setor de Videocirurgia da Disciplina de Técnica Operatória e Cirurgia Experimental (TOCE) da UNIFESP; Professor Orientador do Programa de Pós-Graduação em Cirurgia e Experimentação da UNIFESP; Ex-Professor Visitante da Universidade de Lyon, França; Líder do Grupo de Pesquisa em Videocirurgia do CNPq/UNIFESP

3. Médico-Assistente do Serviço de Gastroenterologia Cirúrgica e Chefe do Setor de Videocirurgia do Hospital do Servidor Público do Estado de São Paulo; Professor Colaborador do Setor de Videocirurgia da TOCE-UNIFESP e do Programa de Pós-Graduação em Cirurgia e Experimentação da UNIFESP; Pesquisador do Grupo de Videocirurgia do CNPq/UNIFESP.

4. Diretor do Serviço de Gastroenterologia Cirúrgica do Hospital do Servidor Público do Estado de São Paulo.

5. Pós-Graduando do Programa de Pós-Graduação em Cirurgia e Experimentação da UNIFESP; Pesquisador do Grupo de Videocirurgia do CNPq/ UNIFESP.

6. Médica-Assistente do Serviço de Gastroenterologia Cirúrgica do Hospital do Servidor Público do Estado de São Paulo; Pesquisadora do Grupo de Videocirurgia do CNPq/UNIFESP.

Recebido em 09/05/2005

Aceito para publicação em 09/09/2005

Conflito de interesse: nenhum

Fonte de financiamento: nenhuma

Trabalho realizado no Serviço de Gastroenterologia Cirúrgica do Hospital do Servidor Público do Estado de São Paulo (HSPE) e no Setor de Videocirurgia da Disciplina de Técnica Operatória e Cirurgia Experimental (TOCE), Departamento de Cirurgia da Universidade Federal de São Paulo (UNIFESP), Programa de Pós-Graduação em Cirurgia e Experimentação. 
os vasos ilíacos comuns, são particularmente propensos a ferimentos durante a punção com agulha de Veress nas proximidades da cicatriz umbilical (Figura 1). Apesar da prevalência dessas ocorrências ser muito baixa $(0,05 \%)$, a mortalidade atinge índices entre $8 \%$ e $17 \%{ }^{8}$.

Todos os ferimentos de grandes vasos por agulha de Veress relatadas na literatura foram causados por punções na linha média, realizadas nas proximidades da cicatriz umbili$\mathrm{cal}^{9}$. O risco da ocorrência desse tipo de lesão iatrogênica é minimizado quando as punções são feitas em local afastado da linha média ${ }^{10,11}$.

Adicionalmente, pacientes com cirurgia abdominal prévia têm risco aumentado de lesões viscerais por agulha de Veress por causa de aderências peritoneais, que tipicamente se localizam ao nível da cicatrização da incisão cirúrgica do peritônio parietal anterior. Estudos necroscópicos encontraram aderências em $74 \%$ a $95 \%$ de pacientes com intervenções cirúrgicas abdominais prévias ${ }^{3}$. As incisões medianas são as que apresentam o maior risco de aderências em torno da cicatriz umbilical. Não obstante, mesmo incisões abdominais afastadas do umbigo podem determinar a formação de aderências na região periumbilical ${ }^{3}$.

Por outro lado, a punção no hipocôndrio esquerdo tem sido mencionada como sendo segura, sem risco maior de

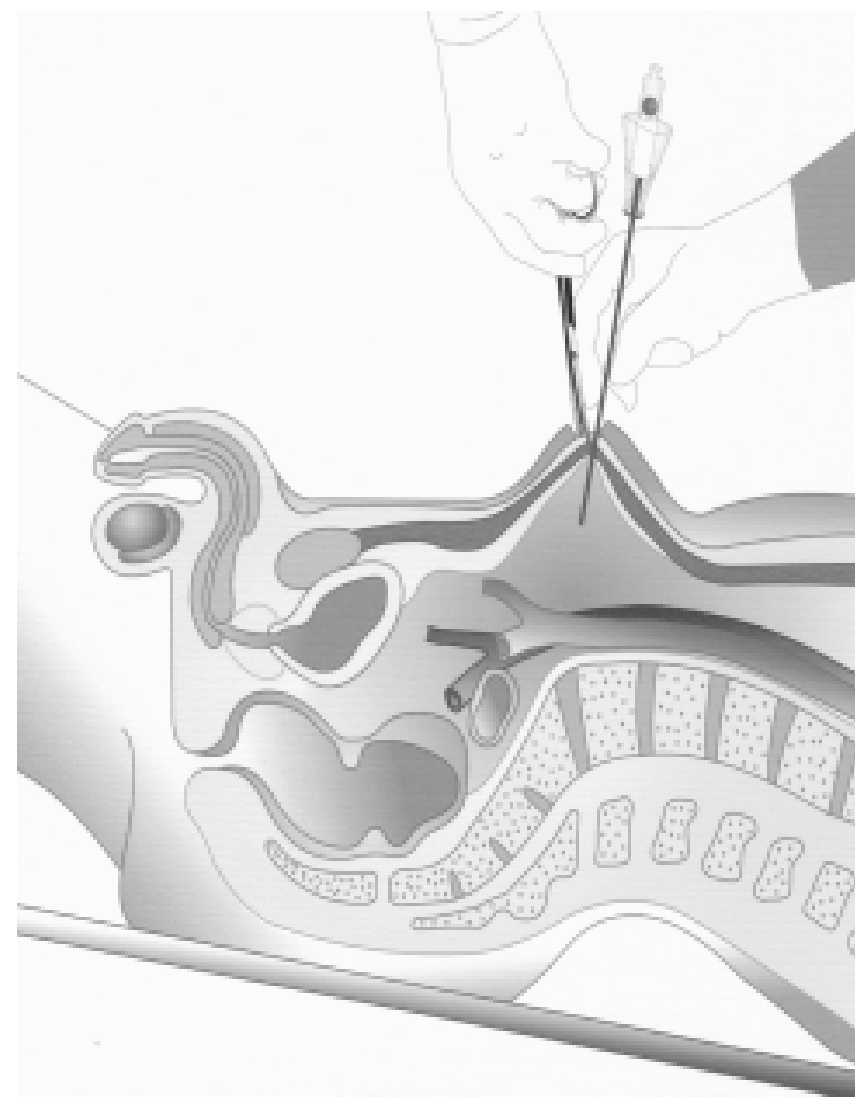

Figura 1 - Esquema representativo da punção com agulha de Veress na linha média do abdome, ao nível da cicatriz umbilical, constituído por corte sagital do abdome e pelve, podendo-se observar a incisão da pele e tela subcutânea, através da qual foi introduzida pinça para tração superior da aponeurose anterior do músculo reto abdominal e introdução da agulha de Veress na cavidade peritoneal, notando-se a proximidade com os grandes vasos retroperitoneais. lesão iatrogênica ${ }^{10-12}$. Tenha-se ainda em conta que a estrutura imediatamente subjacente à parede abdominal anterior no local da punção do hipocôndrio esquerdo é o corpo do estômago que, se acidentalmente lesionado por uma agulha de punção, não necessariamente produzirá escapes de conteúdo, pois a ação da tripla camada muscular de suas paredes tende a ocluir o orifício de punctura. Tenha-se também em conta que tal lesão é de fácil diagnóstico à inspeção inicial da cavidade peritoneal, e passível de ser reparada por laparoscopia mediante um simples ponto de sutura. Por outro lado, no caso de punção acidental do corpo gástrico com insuflação de gás exclusivamente no lume desse órgão, o escape gasoso pela sonda orogástrica será exuberante e denunciará a situação.

Adicionalmente, deve ser considerada a raridade das aderências na região do hipocôndrio esquerdo. É sabido que os movimentos respiratórios do diafragma dificultam o acoplamento das estruturas intra-abdominais à parede abdominal na região do hipocôndrio esquerdo, dessa forma elidindo a formação de aderências peritoneais a esse nível. Por tudo isso a punção no hipocôndrio esquerdo é a preferida por alguns cirurgiões em pacientes obesos e naqueles com laparotomia prévia ${ }^{11,12}$

Entretanto, apesar das vantagens teóricas da punção do hipocôndrio esquerdo em relação à punção na linha média quanto à segurança, não se encontra na literatura nenhum trabalho de comparação entre essas punções. É óbvio que, caso sejam igualmente eficazes, a punção situada no hipocôndrio esquerdo deva ser a preferida, por ser menos perigosa.

O objetivo desta pesquisa é avaliar se a punção com agulha de Veress no hipocôndrio esquerdo, visando à instalação do pneumoperitônio, é uma alternativa segura e eficaz em relação à habitualmente realizada na linha média em região periumbilical.

\section{MÉTODO}

Para a realização de um ensaio clínico de equivalência, prospectivo e randomizado, visando testar a eficácia técnica da criação de pneumoperitônio artificial mediante punção com agulha de Veres no hipocôndrio esquerdo, tendo como termo de comparação a punção realizada na linha média, sessenta e dois pacientes adultos, não-obesos - Índice de Massa Corpórea (IMC) menor do que $30 \mathrm{Kg} / \mathrm{m}^{2}$-, sem história de peritonite pregressa nem prévia violação cirúrgica da cavidade peritoneal foram consecutivamente agendados para serem submetidos a procedimentos videolaparoscópicos no Serviço de Gastroenterologia Cirúrgica do Hospital do Servidor Público do Estado de São Paulo. Os pacientes foram aleatoriamente distribuídos em grupo HE $(n=30)$ - submetidos a punção no hipocôndrio esquerdo -, e grupo LM $(n=32)$, com punção na linha média do abdome, ao nível da cicatriz umbilical.

Esta pesquisa foi aprovada pelo Comitê de Ética em Pesquisa do Instituto de Assistência Médica ao Servidor Público Estadual ( $n^{\circ}$ 045/03) e pelo Comitê de Ética em Pesquisa da Universidade Federal de São Paulo (n 1405/03).

Os grupos foram equivalentes entre si (Tabela 1) no que se refere à idade, sexo, IMC, altura, peso e condição clíni- 
Tabela 1 - Dados demográficos da amostra e comparação entre o grupo de pacientes com punção na linha média (LM) e o grupo com punção no hipocôndrio esquerdo (HE).

\begin{tabular}{|c|c|c|c|}
\hline \multirow[t]{2}{*}{ Parâmetros } & \multicolumn{2}{|c|}{ Técnica } & \multirow[t]{2}{*}{ Comparação } \\
\hline & LM $(n=32)$ & HE $(n=30)$ & \\
\hline \multicolumn{4}{|l|}{ Idade (anos) } \\
\hline média (dp) & $53,2 \quad(12,8)$ & $54,3 \quad(13,6)$ & \\
\hline mínimo-máximo & $31-77$ & $27-77$ & $\mathrm{p}_{1}=0,748$ \\
\hline \multicolumn{4}{|l|}{ Sexo $-\mathrm{n}(\%)$} \\
\hline feminino & $(59,4)$ & $(56,7)$ & \\
\hline masculino & $(40,6)$ & $(43,3)$ & $\mathrm{p}_{2}=0,829$ \\
\hline \multicolumn{4}{|l|}{ IMC $\left(\mathrm{kg} / \mathrm{m}^{2}\right)$} \\
\hline média (dp) & $25,4 \quad(2,4)$ & $25,4 \quad(2,5)$ & \\
\hline mínimo-máximo & $20,6-29,4$ & $21,0-29,7$ & $\mathrm{p}_{1}=0,969$ \\
\hline \multicolumn{4}{|l|}{ Altura (m) } \\
\hline média (dp) & $1,64 \quad(0,10)$ & $1,64 \quad(0,08)$ & \\
\hline mínimo-máximo & $1,45-1,87$ & $1,48-1,78$ & $\mathrm{p}_{1}=0,995$ \\
\hline \multicolumn{4}{|l|}{ Peso $(\mathrm{kg})$} \\
\hline média (dp) & $68,8 \quad(11,0)$ & $68,7 \quad(8,7)$ & \\
\hline mínimo-máximo & $49,5-90,0$ & $53,0-89,8$ & $\mathrm{p}_{1}=0,957$ \\
\hline \multicolumn{4}{|l|}{ Condição clínica - n (\%) } \\
\hline colecistopatia crônica & $(59,4)$ & $(76,7)$ & \\
\hline refluxo gastroesofagiano & $(15,6)$ & $(13,3)$ & \\
\hline hérnia inguinal bilateral & $(3,1)$ & $(6,7)$ & $\mathrm{p}_{2}=0,234$ \\
\hline hérnia inguinal direita & $(9,4)$ & $(0,0)$ & \\
\hline hérnia inguinal esquerda & $4 \quad(14,5)$ & $(3,3)$ & \\
\hline
\end{tabular}

dp: desvio padrão; $n$ : número de pacientes; $p_{1}$ : teste $t$ de Student; $p_{2}$ : teste do Qui-quadrado.

ca que motivou a intervenção cirúrgica programada $(\mathrm{p}>0,05)$. A randomização foi feita pelo médico anestesista mediante sorteio com moeda, no sistema de cara ou coroa, com o paciente sob narcose anestésica, imediatamente antes da intervenção cirúrgica.

Foi ministrado 0,1 mg/Kg de midazolam trinta minutos antes do ato anestésico. A indução foi realizada com $2 \mathrm{mg} /$ $\mathrm{Kg}$ de propofol e $0,5 \mathrm{mcg} / \mathrm{Kg}$ de fentanil, e a curarização com $0,5 \mathrm{mg} / \mathrm{kg}$ de atracúrio. Os pacientes foram submetidos à anestesia geral com intubação orotraqueal e ventilação controlada mecânica. Foi introduzida uma sonda orogástrica e aspirado o conteúdo do estômago.

A técnica de punção na linha média consistiu na incisão longitudinal de $12 \mathrm{~mm}$ na pele da região supra-umbilical, com os pacientes colocados na posição de Tredelenburg de aproximadamente 20 graus, seguida da divulsão da tela subcutânea com pinça Kelly até a visibilização da superfície aponeurótica, que foi apreendida com pinça Kocher e tracionada superiormente, elevando-se a parede abdominal. Neste nível introduziu-se obliquamente (20 graus) no plano sagital do abdome uma agulha de Veress em sentido caudal (Figura 1).

$\mathrm{Na}$ técnica de punção no hipocôndrio esquerdo, fezse incisão de $1,2 \mathrm{~mm}$ na pele ao nível do rebordo costal, a cerca de $8 \mathrm{~cm}$ da linha média (quatro traves de dedos - Figura 2), pela qual foi introduzida uma agulha de Veress perpendicularmente à parede abdominal anterior, com os pacientes colocados em posição de proclive de aproximadamente 20 graus.

Nos dois grupos, após a punção foram utilizadas provas preconizadas para testar o posicionamento da agulha no interior da cavidade peritoneal ${ }^{10-12}$ : provas da aspiração, da resistência à infusão, da recuperação do líquido infundido e do escoamento. Adicionalmente, no início da insuflação era feita a prova da pressão inicial.

A prova da aspiração através da agulha de Veress foi considerada positiva quando nenhum material era aspirado com seringa. Na prova da infusão injetava-se $5 \mathrm{ml}$ de solução fisiológica através da agulha, verificando-se moderada resistência ao fluir do líquido (prova positiva) ou, ao contrário, constatando-se aumento dessa resistência (prova negativa). $\mathrm{Na}$ sequiência, aspirava-se a agulha, considerando-se positiva a prova de recuperação na ausência de recobramento do líquido infundido. Na prova do escoamento, após a colocação de $2 \mathrm{ml}$ de solução fisiológica no interior da agulha de Veress a prova era considerada positiva no caso do desaparecimento do líquido após desconexão da seringa. No momento em que qualquer dessas provas era considerada negativa, o procedimento inteiro era abortado, registrando-se os eventos na rubrica de fracasso da punção, sendo todo o processo reiniciado.

A infusão de gás carbônico sob pressão na cavidade peritoneal era iniciada quando as quatro provas descritas fossem todas positivas. A pressão intraperitoneal máxima programada foi de $12 \mathrm{mmHg}$ e o fluxo máximo de um litro por minuto. Caso a pressão intraperitoneal inicial ultrapassasse $8 \mathrm{mmHg}$ durante os 5 segundos iniciais da insuflação, a prova da pressão inicial era considerada negativa, todo o procedimento era abortado e o malogro registrado.

$\mathrm{Na}$ vigência de positividade das provas, prosseguia-se com a insuflação de gás carbônico, registrando-se periodicamen- 


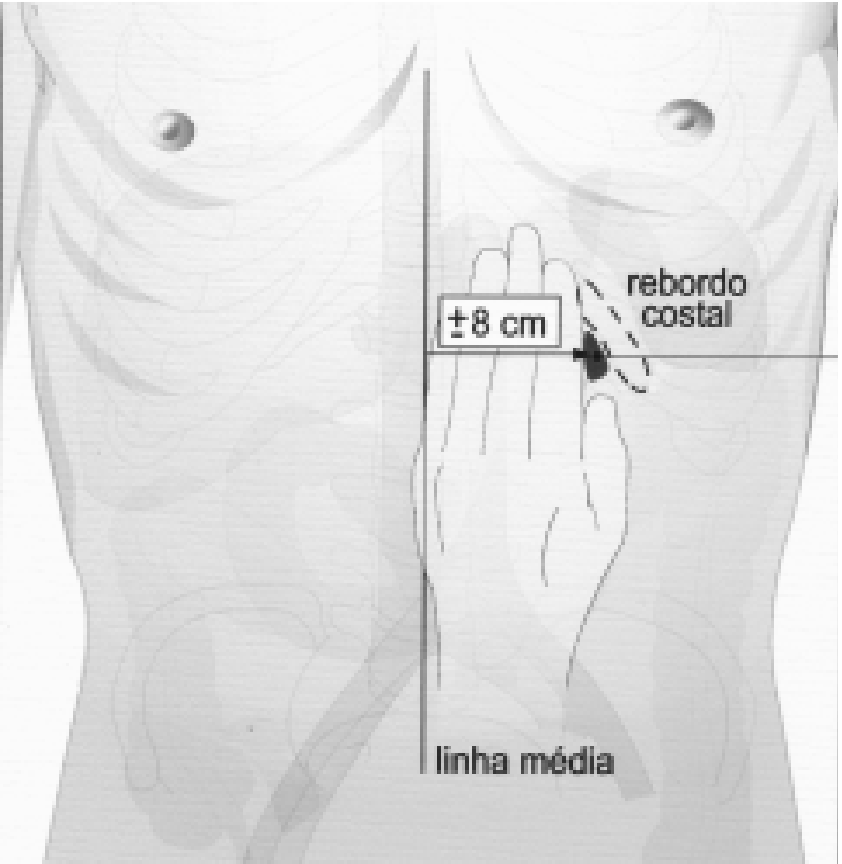

Figura 2 - Esquema representativo da localização da punção com agulha de Veress no hipocôndrio esquerdo, junto ao rebordo costal, a 8 cm da linha média. Observa-se o ponto em que deve ser realizada a punção, notando-se o distanciamento dos grandes vasos retroperitoneais.

te as variações da pressão intraperitoneal, do fluxo corrente e do montante do volume injetado a partir do momento zero e a cada vinte segundos seguintes, até que a pressão intraperitoneal máxima programada $(12 \mathrm{mmHg})$ fosse atingida, ocasião em que o tempo total de duração da insuflação era registrado.

Os dados foram tratados por análises estatísticas. As variáveis qualitativas foram representadas por freqüência absoluta e relativa e as quantitativas por média, desvio padrão e valores mínimos e máximos. A homogeneidade entre os grupos de estudo foi avaliada pelo teste $t$ de Student ou pelo teste do Qui-quadrado, quando indicados. O nível de significância adotado foi de $0,05(\mathrm{a}=5 \%)$. A equivalência entre os grupos de estudo em relação aos parâmetros intra-operatórios e pós-operatórios foi estabelecida pela sobreposição dos intervalos de confiança $\left(\mathrm{IC}_{95 \%}\right)$ construídos para cada parâmetro avaliado dentro de cada grupo. Os intervalos com nível de $95 \%$ de confiança para médias e proporções foram construídos pelas fórmulas padrão para estimadores com distribuição normal. Para avaliar a distribuição dos parâmetros pressão, volume e fluxo em função do tempo foram estimados modelos de regressão polinomiais de $1^{\circ}, 2^{\circ}$ e $3^{\circ}$ graus e o melhor grau de ajuste determinado por análise de resíduos e do coeficiente de explicação $\left(\mathrm{R}^{2}\right)$. Foram construídos intervalos com nível de $95 \%$ de confiança para cada coeficiente estimado pelo modelo e os dois grupos de estudo foram comparados em relação a esses intervalos.

\section{RESULTADOS}

Nos casos de malogros na colocação da ponta da agulha no interior da cavidade peritoneal detectados por meio de cada uma das provas realizadas, os intervalos de confiança verificados nos dois grupos se sobrepuseram e, dessa forma, houve evidência estatística de que são equivalentes quanto às proporções de fracassos encontradas nas diferentes provas.

Quanto à duração da instalação do pneumoperitônio, constatou-se que não houve evidência estatística entre as médias de tempo para atingir a pressão de $12 \mathrm{mmHg}$, em função da sobreposição dos intervalos de confiança dos dois grupos.

No que se refere aos valores das variáveis dependentes (pressão intraperitoneal, fluxo efetivo e montante do volume injetado) ao longo do tempo de insuflação do pneumoperitônio, devido à sobreposição dos intervalos de confiança e dos coeficientes dos modelos estimados para cada grupo avaliado não houve evidência estatística de diferença significante entre os grupos quanto às curvas de regressão da pressão intraperitoneal, do fluxo efetivo e do montante do volume injetado. Tampouco houve diferença estatisticamente significante quanto às médias de área sob as curvas da pressão, do fluxo e do volume.

\section{DISCUSSÃO}

Durante as duas últimas décadas, rápidos avanços na cirurgia videolaparoscópica a fizeram método consagrado. Entretanto, por ser recente, esse tipo de acesso cirúrgico ainda enseja controvérsias. Uma delas diz respeito ao melhor método para o estabelecimento do pneumoperitônio, que pode ser realizado mediante minilaparotomia e introdução de um trocarte comum de laparoscopia ${ }^{13}$ ou do trocarte especial de Hasson ${ }^{14}$. Alternativamente, pode-se inserir às cegas um trocarte óptico especial ${ }^{15,16}$, ou proceder-se à punção da cavidade peritoneal com a agulha de Veress na linha média do abdome ${ }^{7}$, sendo essa última a técnica mais freqüentemente utilizada ${ }^{2,6,17}$.

A criação do pneumoperitônio por punção com agulha de Veress é uma técnica fácil, rápida e efetiva. O problema do método que utiliza a agulha de Veress é a segurança do procedimento. Os principais locais de punção com agulha de Veress são a região umbilical, considerada como padrão ${ }^{7}$, a margem subcostal esquerda ${ }^{10}$, um ponto intermediário entre a borda anterior da sínfise púbica e a cicatriz umbilical ${ }^{18}$, e um ponto idêntico ao de McBurney, na fossa ilíaca esquerda ${ }^{7}$. Há, ainda, relatos de acesso à cavidade peritoneal mediante transfixação do fundo uterino com a agulha introduzida no canal cervical ${ }^{19}$ e também por meio da transfixação da parede da região tóraco-abdominal através do nono espaço intercostal esquerdo ${ }^{20}$

A preocupação com a segurança na punção às cegas com agulha de Veress na linha média, vem sendo freqüentemente motivo de estudos ${ }^{19,21}$. Palmer ${ }^{10}$ descreveu a punção no hipocôndrio esquerdo com agulha de Veress a $3 \mathrm{~cm}$ abaixo do rebordo costal, na linha hemiclavicular. Desde então a técnica sofreu algumas pequenas modificações, com o objetivo de se afastar dos grandes vasos e de aderências na linha média. A punção, neste trabalho, é feita junto ao rebordo costal e a $8 \mathrm{~cm}$ da linha média, o que evita atingir a aorta, a veia cava e os vasos epigástricos superiores. Nessa região a punção é facilitada por ser o fixo o peritônio ao rebordo costal.

Schwartz et al..$^{12}$ induziram pneumoperitônio em 600 pacientes obesos mórbidos utilizando a punção com agulha de 
Veress no hipocôndrio esquerdo. Ocorreu uma lesão puntiforme na camada muscular do cólon transverso, que foi reparada por laparoscopia, com um ponto simples. Não ocorreu perfuração de outras vísceras ocas, sangramento anormal de parede abdominal ou visceral, nem lesão hepática ou esplênica. Rothagi et $a l .{ }^{11}$ realizaram 344 punções no hipocôndrio esquerdo com apenas dois fracassos devido à agulha não ter se alocado na cavidade peritoneal. Aúnica complicação foi um hematoma no grande omento, tratado com conduta expectante. Os autores concluíram que a punção no hipocôndrio esquerdo é efetiva para o estabelecimento do pneumoperitônio.

Alguns cirurgiões, habituados à punção na linha média do abdome, questionam a punção no hipocôndrio esquerdo, argumentando que essa seria mais difícil e necessitaria um maior número de tentativas para ser efetuada. Este trabalho mostrou que, quando comparadas, há evidências estatísticas de que as técnicas são equivalentes quanto às proporções de sucessos e fracassos nas tentativas de se estabelecer pneumoperitônio, encontradas nos diferentes grupos. Os grupos foram equivalentes tanto no número de tentativas quanto em relação à positividade das provas, evidenciando que as dificuldades técnicas entre as punções são semelhantes.

Outro ponto obscuro era quanto à similaridade ou não, entre os dois grupos, do tempo necessário para a instalação do pneumoperitônio, e se as variações de pressão, fluxo e volume se comportariam de modo semelhante. Sendo a agulha colocada em locais diferentes no abdome, com a ocorrência de variações na anatomia topográfica, poderia, teoricamente, haver discrepâncias nos parâmetros avaliados. Os resultados desta pesquisa mostraram que os grupos se comportaram de forma equivalente no que diz respeito às variações de todos esses parâmetros.

Quanto às discussões teóricas sobre as possibilidades de iatrogenias com punção no hipocôndrio esquerdo, este trabalho não foi capaz de revelar conclusões definitivas, pois essas lesões são muito raras em mãos experientes.

Permanecem as ponderações de que o hipocôndrio esquerdo não é território de grandes vasos, podendo-se praticamente descartar lesões dos mesmos com a punção sendo feita nessa região. Adicionalmente, deve-se considerar que os cólons e o intestino delgado não se encontram na projeção do local da punção no hipocôndrio esquerdo, e nem o baço ou o fígado de dimensões normais encontram-se sob o rebordo costal esquerdo. Por outro lado, o estômago e os omentos são subjacentes ao local da punção, podendo ser eventualmente lesados. Entretanto, tais lesões não se configuram como sendo de maior gravidade.

As aderências no hipocôndrio esquerdo são, ao contrário do que ocorre na região umbilical, muito raras, entretanto podendo estar presentes em pacientes com antecedentes de esplenectomia ou de colectomias que necessitem mobilização do ângulo esplênico do cólon. Nesses pacientes é de bom senso que não se proceder à punção no hipocôndrio esquerdo.

Para estimar a frequiência de aderências peritoneais e viscerais à região umbilical de acordo com história pregressa de cirurgia, Audebert e Gomel ${ }^{22}$ realizaram estudo prospectivo em 814 pacientes. A realização de laparoscopia mediante laparoscópico de $1,2 \mathrm{~mm}$ de diâmetro introduzido no hipocôndrio esquerdo permitiu atestar a presença de aderências peritoneais ao nível da região umbilical, dessa forma elidindo o risco de lesão intestinal pela inserção inadvertida do primeiro trocarte nessa região. Separando os pacientes em grupos, a incidência de aderências e a determinação do risco foram $0,68 \%$ e $42 \%$ entre 469 pacientes sem cirurgia prévia, $1,6 \%$ e $0,8 \%$ entre 125 pacientes com cirurgia laparoscópica prévia, 19,8\% e 6,8\% dentre 131 pacientes com laparotomia supra-púbica horizontal e $51,7 \%$ e $31,4 \%$ dentre 89 pacientes com laparotomia mediana.

Levrant el al..$^{23}$ também comprovaram que uma laparotomia mediana vertical aumenta o risco de aderências na parede abdominal anterior em comparação a incisões abdominais transversais.

Dessa forma, a punção com agulha de Veress no hipocôndrio esquerdo para o estabelecimento do pneumoperitônio é uma alternativa segura e eficaz, devendo ser a preferida.

\section{AGRADECIMENTOS}

Agradecemos os equipamentos e materiais laparoscópicos, que nos foram cedidos pela PCE - Pró-Cirurgia Especializada Ltda.

\begin{abstract}
Background: In the creation of the pneumoperitoneum with the Veress neddle, all reported incidents occurred during the puncture in the midline of the abdomen. Albeit rare, such type of incident has a high mortality rate as it normally severs the large vessels. The objective of this research is to test the efficacy of a safer alternative method of puncture in the left hipocondrium. Methods: Sixty two patients, distributed randomly into two groups were studied. Group HE: puncture in the left hipocondrium ( $n=30)$, and Group LM: puncture in the medline of the abdomen $(n=32)$. An evaluation was carried out of the results of the tests as to the positioning of the needle, the number of failed attempts to access the peritoneal cavity and the time taken for the creation of the pneumoperitoneum. Real flows, intraperitoneal pressure, and injected volumes were recorded at 20 second intervals, until pressure had reached $12 \mathrm{mmHg}$. Results: The results of the test regarding the positioning of the Needle were equally positive. There were two unsuccessful attempts at piercing in Group LM and one in Group HE. The time taken for the creation of the pneumoperitoneum was on average of 3 minutes and 46 seconds for Group HE and of 4 minutes and 2 seconds for Group LM. The average measures recorded regarding flow, pressure and volume were equivalent in each Group, respectively. The statistical analysis has demonstrated that piercing of the left hypocondrium was as effective in the creation of the pneumoperitoneum as it was the case as regards the piercing of the medium line of the abdomen. Conclusion: The left hipocondrium must be the preferred place for the puncture with the Veress needle so as to create the artificial pneumoperitoneum by the closed technique, as it entails a smaller risk.
\end{abstract}

Key Words: Laparoscopy/ adverse effects; Surgical procedures, Operative; Pneumoperitoneum, Artificial; Biopsy, Needle; Biopsy, Needle/ adverse effects. 


\section{REFERÊNCIAS}

1. Mouret PH. Cirurgia laparoscópica: una evolución de la filosofia quirúrgica? In: Mineiro M, Melotti G, Mouret PH, editores. Cirurgía laparoscópica. Madrid: Panamericana; 1996. p. 1-12.

2. Catarci M, Carlini M, Gentileschi P, et al. Major and minor injuries during the creation of pneumoperitoneum. A multicenter study on 12,919 cases. Surg Endosc. 2001;15(6):566-9.

3. Anaise D. Vascular and bowel injuries during laparoscopy. Available from: http://www.danaise.com/ vascular and bowel injuries duri.htm

4. Neudecker J, Sauerland S, Neugebauer E, et al. The European Association for Endoscopic Surgery clinical practice guideline on the pneumoperitoneum for laparoscopic surgery. Surg Endosc. 2002;16(7):1121-43.

5. Veress J. [Neues Instument zur Ausfuhrung von Brust-oder Bauchpunktionen und Pneumothoraxbehandlung]. Dtsch Med Wochenshr. 1938;41:1480-1.

6. Molloy D, Kaloo PD, Cooper M, et al. Laparoscopic entry: a literature review and analysis of techniques and complications of primary port entry. Aust N Z J Obstet Gynaecol. 2002;429(3):246-53.

7. Guimarães P. Pneumoperitônio, punções e trocartes. In: Donadio $\mathrm{N}$, Albuquerque Neto LC, editores. Consenso Brasileiro em Videoendoscopia Ginecológica. São Paulo: Artes Médicas; 2001. p. 27-32.

8. Roviaro GC, Varoli F, Saguatti L, et al. Major vascular injuries in laparoscopic surgery. Surg Endosc. 2002;16(8):1192-6.

9. Azevedo JLMC, Saldanha C, Hobi C, et al. Revisão bibliográfica sistemática das lesões causadas pela agulha de Veress durante a criação do pneumoperitônio. In press

10. Palmer R. Safety in laparoscopy. J Reprod Med 1974;13(1):1-5.

11. Rohatgi A, Widdison AL. Left subcostal closed (Veress needle) approach is a safe method for creating a pneumoperitoneum. J Laparoendosc Adv Surg Tech A. 2004;14(5):278-80.

12. Schwartz ML, Drew RL, Andersen JN. Induction of pneumoperitoneum in morbidly obese patients. Obes Surg. 2003;13(4):601-4.

13. Dingfelder JR. Direct laparoscope trocar insertion without prior pneumoperitoneum. J Reprod Med. 1978;21(1):45-7.

14. Hasson HM. A modifield instrument and method for laparoscopy. Am J Obstet Gynecol. 1971;110(6):886-7
15. String A, Berber E, Foroutani A, et al. Use of optical access trocar for safe and rapid entry in various laparoscopic procedures. Surg Endosc. 2001;15(6):570-3.

16. Bemelman WA, Dunker MS, Busch OR, et al. Efficacy of establishment of the pneumoperitoneum with the Veress needle, Hasson trocar, and modified blunt trocar (TrocDoc): a randomized study. J Laproendosc Adv Surg Tech A. 2000;10:325-30.

17. Sandor J, Ballagi F, Nagy A, et al. A needle-puncture that helped to change the world of surgery. Homage to Janos Veres. Surg Endosc. 2000;14(2):201-2.

18. Lee CL, Huang KG, Jain S, et al. A new portal for gynecologic laparoscopy. J Am Assoc Gynecol Laparosc. 2001;8(1):147-50

19. Santala M, Jarvela I, Kauppila A. Transfundal insertion of a Veress needle in laparoscopy of obese subjects: a practical alternative. Hum Reprod. 1999;14(9):2277-8.

20. Childers JM, Brzechffa PR, Surwit EA. Laparoscopy using the left upper quadrant as the primary trocar site. Gynecol Oncol. 1993;50(2):221-5.

21. Ostrzenski A. Randomized, prospective, single-blind trial of a new parallel technique of Veress pneumoperitoneum needle insertion versus the conventional closed method. Fertil Steril. 1999;71(3):578-81.

22. Audebert AJ, Gomel V. Role of microlaparoscopy in the diagnosis of peritoneal and visceral adhesions and in the prevention of bowel injury associated whith blind trocar insertion. Fertil Steril. 2000;73(3):631-5.

23. Levrant SG, Bieber E, Barnes R. Risk of anterior abdominal wall adhesions Increase whith number and type of previous laparotomy. J Am Assoc Gynecol Laparosc. 1994;1(4,Part 2):S19.

Endereço para correspondência:

Prof Dr João Luiz M. C. Azevedo

Universidade Federal de São Paulo

Setor de Videocirurgia

Prédio da Cirurgia Experimental.

Rua Botucatu, 740 - V. Clementino

CEP 04023-900, São Paulo.SP.

Telfax: 11 5576-4272

E-mail: jozevedo.dcir@epm.br

Site: http://www.cirurgiaonline.med.br 